\title{
Experimental Analysis of Beam Pointing System Based on Liquid Crystal Optical Phase Array
}

\author{
Yubin SHI", Jianmin ZHANG, and Zhen ZHANG \\ State Key Laboratory of Laser Interaction with Matter, Northwest Institute of Nuclear Technology, Xi'an, 710024, China \\ ${ }^{*}$ Corresponding author: Yubin SHI $\quad$ E-mail: shiyubin@nint.ac.cn
}

\begin{abstract}
In this paper, we propose and demonstrate an elementary non-mechanical beam aiming and steering system with a single liquid crystal optical phase array (LC-OPA) and charge-coupled device (CCD). With the conventional method of beam steering control, the LC-OPA device can realize one dimensional beam steering continuously. An improved beam steering strategy is applied to realize two dimensional beam steering with a single LC-OPA. The whole beam aiming and steering system, including an LC-OPA and a retroreflective target, is controlled by the monitor. We test the feasibility of beam steering strategy both in one dimension and in two dimension at first, then the whole system is build up based on the improved strategy. The experimental results show that the max experimental pointing error is $56 \mu \mathrm{rad}$, and the average pointing error of the system is $19 \mu \mathrm{rad}$.
\end{abstract}

Keywords: Lasers and laser optics; laser beam shaping; liquid crystals; optical engineering

Citation: Yubin SHI, Jianmin ZHANG, and Zhen ZHANG, "Experimental Analysis of Beam Pointing System Based on Liquid Crystal Optical Phase Array," Photonic Sensors, 2016, 6(4): 289-294.

\section{Introduction}

Laser acquisition, tracking, pointing (ATP) system has played an important role in free space optical communication, laser radar, and other applications for a long while [1-3]. It is well known that traditional ATP systems based on the mechanical mirrors and gimbals are complex and expensive, with relatively large volumes and high weight. For the traditional ATP systems, beam steering and stabilizing are still major limitations [4]. However, optical phased array techniques can avoid these problems effectively. In recent years, liquid crystal optical phased array (LC-OPA) techniques have been widely developed and have been considered to be potential in several applications such as laser steering, tracking, and optical tweezers [5-8].
Methods of improving LC-OPA beam steering efficiency, steering angle, and pointing (steering) accuracy have been reported [9-11], however, little work has been done to test and verify the steering performances of LC-OPA in a beam steering system.

In this paper, we present a beam steering system with a single LC-OPA device, which can realize two-dimensional continuous beam steering within a max steering angle. This paper is organized as follows. Firstly, the experimental setup is introduced. Secondly, the conventional and improved beam steering strategy of LC-OPA is described. Then, a numerical simulation of two dimensional beam steering and a beam pointing experiment based on LC-OPA are reported, and the steering error is analyzed. Finally, the whole system based on the steering strategy is established, and the performance of the system is measured.

Received: 24 May 2016 / Revised: 3 June 2016

(C) The Author(s) 2016. This article is published with open access at Springerlink.com

DOI: $10.1007 / \mathrm{s} 13320-016-0348-2$

Article type: Regular 
The schematic diagram for beam aiming and pointing system is shown in Fig. 1. The essential instruments are LC-OPA (BNS Company, LC-OPA with $256 \times 25624 \mu \mathrm{m} \times 24 \mu \mathrm{m}$ pixels). The light source used is a $1064-\mathrm{nm} \mathrm{Nd}: \mathrm{YVO}_{4}$ polarized laser beam. A telescope is employed for beam expanding and collimating. A polarized beam splitter (PBS1), a half-wave plate, and a polarized beam splitter (PBS2) are used together to provide the LC-OPA with s-linearly polarized laser beam, which makes it possible to adjust energy of the laser. Beam splitter (BS1) is utilized for the beam transmitted from the LC-OPA vertically. The emission beam is separated by the beam splitter (BS2) in two parts. Most of the beam is reflected to the target in far field. The last part is incident on a retro-reflector and then focused on the charge-coupled device (CCD) by a lens with 12.5-mm focal length. The CCD camera (Dolphin F-145B, $15 \mathrm{~Hz})$ with $1280 \times 9606.45 \mu \mathrm{m} \times 6.45 \mu \mathrm{m}$ pixels is used to diagnose the far-field intensity distribution of the output beam and the far-field target. Since we hope that the beam efficiency is high in our system, the splitting ratio (index of transmission: index of reflection) of BS1 should be 5:5 to guarantee the maximum energy of beam, and the reflection of the BS2 should be close to 1 (the beam still need to be transmitted as a small signal). In the experiment, the splitting ratio (index of transmission: index of reflection) of the BS1 is 44:56, and the splitting ratio of the BS2 is 84:16.

The CCD camera is directly interfaced by using an IEEE 1394 (Firewire) connection to computer. With the CCD detector and the retro-reflector, the position information between the target and the laser is acquired, and then the tilt phase distributions which loaded on LC-OPA can be calculated by the computer. This experiment is done at an indoor laboratory facility, and the distance between the BS2 and the target is approximately $2 \mathrm{~m}$.

The beam steering method is verified, and the beam steering accuracy is measured with CCD image system in front of the BS2. On the basis of the results, the beam pointing experiment is done further.

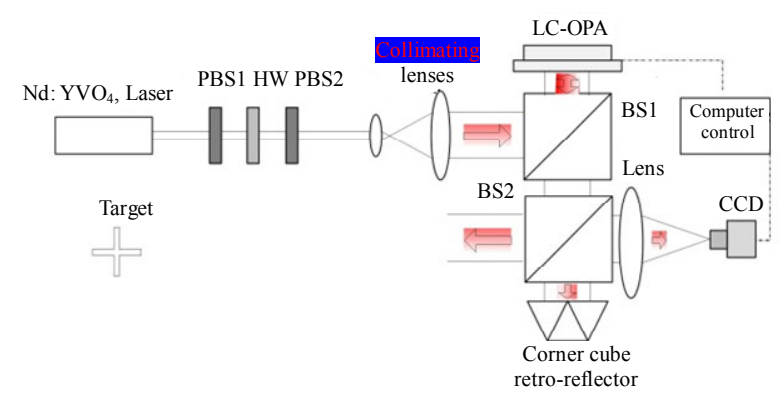

Fig. 1. Schematic diagram of laser aiming and pointing experiment system based on LC-OPA. Both BS1 and BS2 are the non-polarization beam splitters, and BS1 is used to ensure that the transmitted beam falls on LC-OPA perpendicularly, and $\mathrm{BS} 2$ is used to ensure that part of the beam enters into CCD.

According to the conventional method, when the phase modulation of LC-OPA is periodic, the steering angle of the incident beam can be given by [5]

$$
\theta=\arcsin (\lambda / N d)
$$

where $\theta$ is the deflection angle, $\lambda$ is the working wavelength, $N$ is the number of phase shifters (or called pixels) in one period, and $d$ is the size of a single phase shifter. In addition, the largest realizable deflection angle $\theta_{\max }$ is $\arcsin (\lambda / 2 d)$. However, periodic phase modulation of LCOPA cannot realize continuous beam deflection angle within $\pm \theta_{\max }$. Based on the theory above, Engström provided a non-periodic method to realize one dimensional continuous beam steering strategy in the first part [9], which can be summarized as:

$$
\phi_{j}(\theta)=\operatorname{round}\left[\phi_{j, \text { ideal }}(\theta) \cdot M / 2 \pi\right] \cdot 2 \pi / M
$$

where $\phi_{j \text {, ideal }}$ is the ideal staircase phase related to the deflection angle of $j$ pixel, $j=1,2, \cdots, N$, and $N$ is the total number of pixels. $M$ is the equidistant phase level between 0 and $2 \pi$, and round simply round the value to the closest integer value. From(2), two dimensional (2D) beam steering method can be derived further:

$$
\phi_{j, \text { final }}=\operatorname{imrotate}\left[\phi_{j}(\theta), \Theta\right]
$$

where imrotate represents rotate $\phi_{j}$ with angle $\Theta$ conter-clockwise, $\Theta$ is arbitrary number between 0 
and $2 \pi$, and $\phi_{j \text {, final }}$ is the modulated phase for $j$ pixel for $2 \mathrm{D}$ beam steering.

Compared with the conventional 2D beam steering strategy, elevation angle $\alpha$ and azimuth angle $\beta$ are always used to describe the deflection angle. Here the relation between $(\theta, \Theta)$ and $(\alpha, \beta)$ is summarized as follows:

$$
\theta=\arctan \left[\sqrt{\tan ^{2}(\alpha)+\tan ^{2}(\beta)}\right]
$$

and

$$
\Theta=\operatorname{arccot}[\tan (\beta) / \tan (\alpha)] .
$$

The numerical simulation results are done with pixel size $24 \mu \mathrm{m}$ and wavelength of $1064 \mathrm{~nm}$. The waist of the Gauss beam is $0.3 \mathrm{~mm}$, and the focus of the lens is $0.1 \mathrm{~m}$. We assume there are no fringing fields degrading the performance in the numerical simulation.

The numerical results show the relationship between the aiming angle and the realized angle in Fig. 2. There are some fluctuations in the linear relationship between the aiming angle and steering angle, and this phenomenon is inevitable due to the quantized staircase phase of an LC-OPA. We call this error as a theoretical error. The zeroth diffraction order is due to the unity fill factor of pixels in an LC-OPA device (active area of the pixel is smaller than the separation of pixels). Furthermore, the normalized steering error is conventionally defined as

$$
\varepsilon=\left|\theta-\theta_{\text {ideal }}\right| / \theta_{\text {spot }}
$$

where $\theta_{\text {ideal }}$ is the aiming angle, $\theta$ is the realized steering angle, and $\theta_{\text {spot }}$ is the diffraction limited spot size of the beam [6]. The experimental steering error at first is given in Fig. 3. As shown in Fig. 3, the steering error is symmetric around $0 \mathrm{mrad}$, and it appears that the steering error is periodic within the max steering angle. Also, it is obvious that the fluctuation of the error become bigger as the steering angle becomes larger. It suggests that we had better use the beam steering strategy in middle of the field of view (FOV) in a beam steering system.
Theoretically, the pointing error can be explained as the results of quantized staircase phase of an LC-OPA and fringe effect. However, phase aberration in the system influences the steering accuracy of the system, especially phase tilt. The results also infer that the strategy is relatively stable in a small FOV.

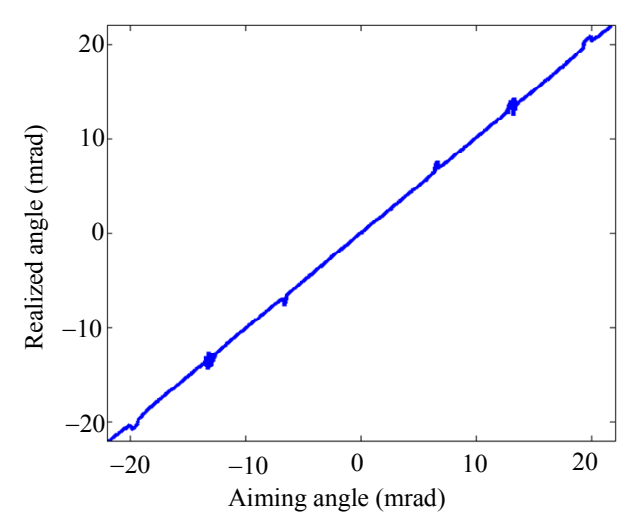

Fig. 2 Numerical simulation result of relationship between realized angle and steering angle.

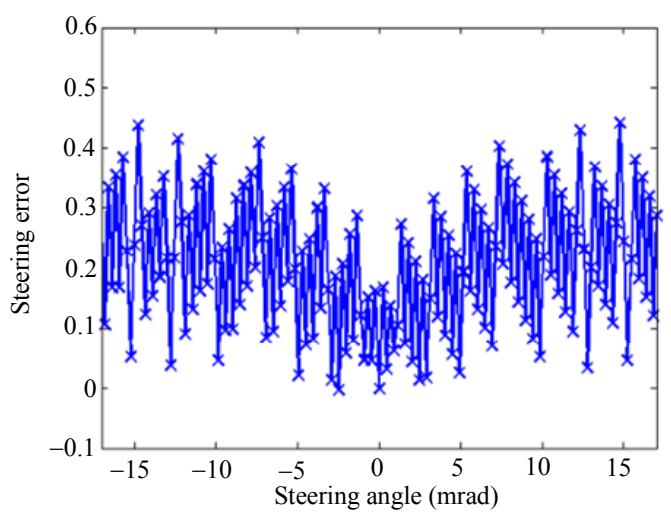

Fig. 3 Experimental result of relationship between beam steering error and the steering angle.

Figure 4 shows the results of two-dimensional beam steering. Four different values of $\Theta$ are given, when $\theta$ equals $10 \mathrm{mrad}$. The original beam is deflected to different directions. The results show that the 2D beam strategy is feasible. However, there are several diffraction orders. It can be attributed to the non-unity fill factor of spatial light modulator (SLM).

We also define the beam steering errors of $\Theta$ as

$$
\gamma=\left(\Theta-\Theta_{\text {ideal }}\right) / \theta_{\text {spot }}
$$

where $\Theta_{\text {ideal }}$ is the desired steering angles, and $\Theta$ is 
the realized steering angle. The beam steering error of $\Theta$ is shown in Fig. 5. It shows that the steering error $\gamma$ is bigger as the steering angle becomes bigger.
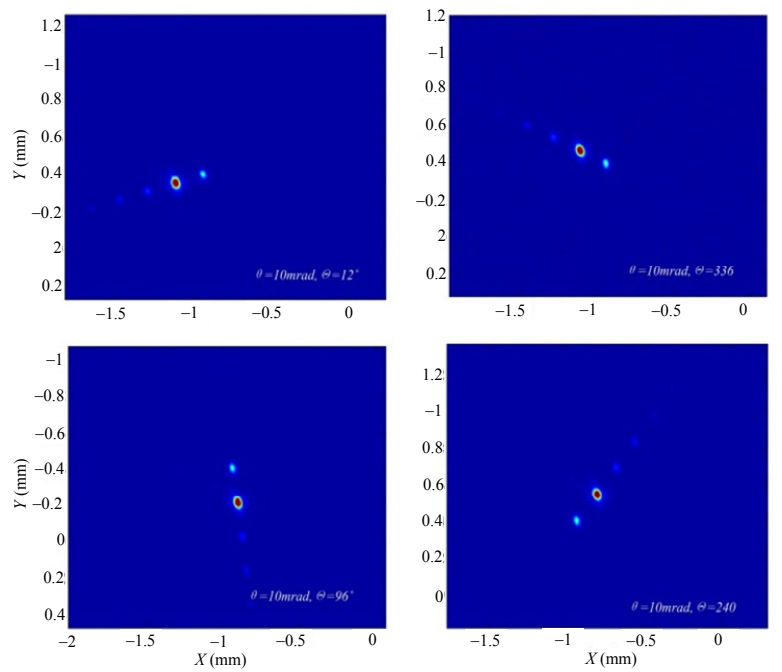

Fig. 4 Experimental results of $2 \mathrm{D}$ beam steering with $\theta=$ $10 \mathrm{mrad}$.

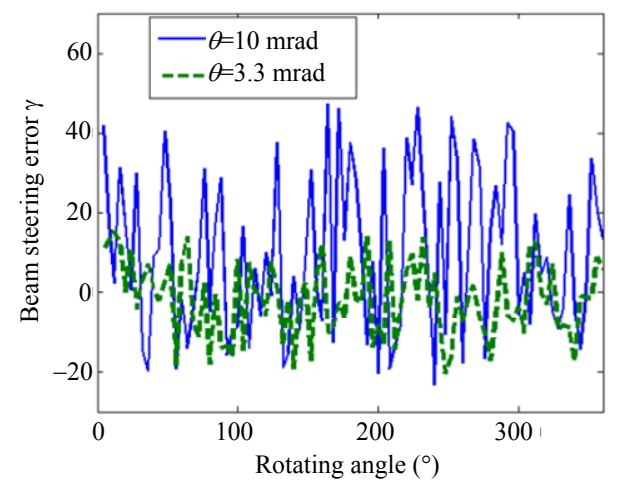

Fig. 5 Steering error of $2 \mathrm{D}$ beam steering with $\theta=10 \mathrm{mrad}$ and $\theta=3.3 \mathrm{mrad}$.

After verifying the feasibility of the $2 \mathrm{D}$ beam steering method, we combine the $2 \mathrm{D}$ beam steering strategy and the LC-OPA together in order to realize beam aiming and steering in an electro-optical system.

The images of retroreflective target are shown in Fig. 6. In order to evaluate the aiming and pointing error, a cross target about $5 \mathrm{~mm}$ wide and $5 \mathrm{~mm}$ high is used. When the target is illumined by light, the target can be acquired by the CCD camera and the image is shown in Fig. 6(a). When it is illumined by the transmitted laser, the image of the target is shown in Fig. 6(b).

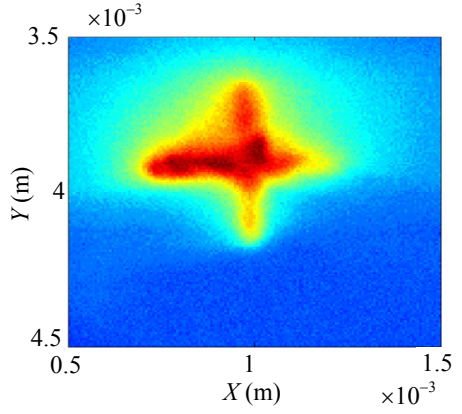

(a)

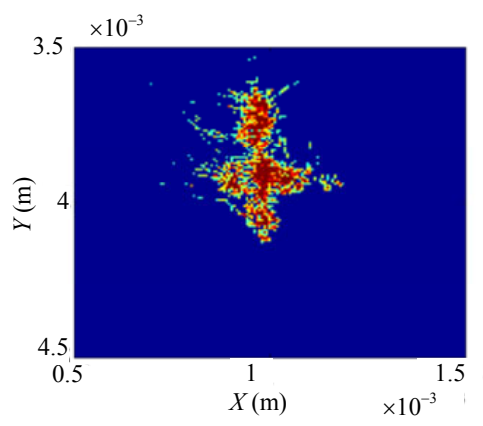

(b)

Fig. 6 Images of cross target: (a) image of target without lasers illuminating on it and (b) image of target with lasers illuminating on it ( the background noise has been removed).

When the target is moved by the stepper motor in two directions, the centroid of target can be calculated from the target image. If we adjust the corner cube retro-reflector properly, the beam which is incident on it can be regarded as a beacon to calculate the steering angle. Then the phase distribution can be calculated further and can be loaded on the LC-OPA to realize beam steering. Here the pointing error is defined as

$$
\begin{aligned}
& \text { error }_{p}= \\
& \left\{\left[\left(x_{\text {retro }}-x_{\text {tar }}\right)^{2}+\left(y_{\text {retro }}-y_{\text {tar }}\right)^{2}\right]^{0.5} \cdot d_{\mathrm{CCD}}\right\} / L
\end{aligned}
$$

where $x_{\text {tar }}$ and $y_{\text {tar }}$ are the centroid coordinates of the target, $x_{\text {retro }}$ and $y_{\text {retro }}$ are the centroid coordinates of the retro-reflect beam, $L$ is the distance between the target and BS2, and $d_{\mathrm{CCD}}$ is the pixel size of the CCD. When the target is moving mainly along horizontal direction and vertical direction, the results are recorded and shown in Figs. 7 and 8.

Compare Fig. 7(a) with Fig.7(b), we can see that the target is moving along $X$ axis (horizontal) from the variation of vertical coordinate. Even though the 
target is moving along one single direction, another direction will produce jitter in fact. The reason why this happens can be ascribed to two main points. Firstly, the 1D beam steering method has errors between the aiming angle and the realized angle in theory. Secondly, influence factors cannot be avoided in the experiment, such as the target position, target shape, et al. As a result of what have been discussed above, changes in both directions should be taken into consideration, and both will cause pointing error in the system. From these result, if the centroid of the retro-reflect beam is not zero, it shows that the beam steers to the target. But there is difference between the centroid of the target and the retro-reflect beam. In order to minimize the pointing error in this kind of evaluation criterion, we try our best to make the size of the target close to the far field spot size.

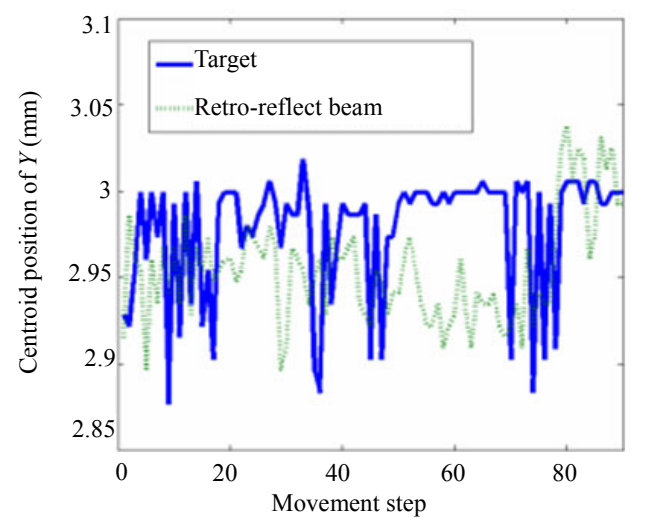

(a)

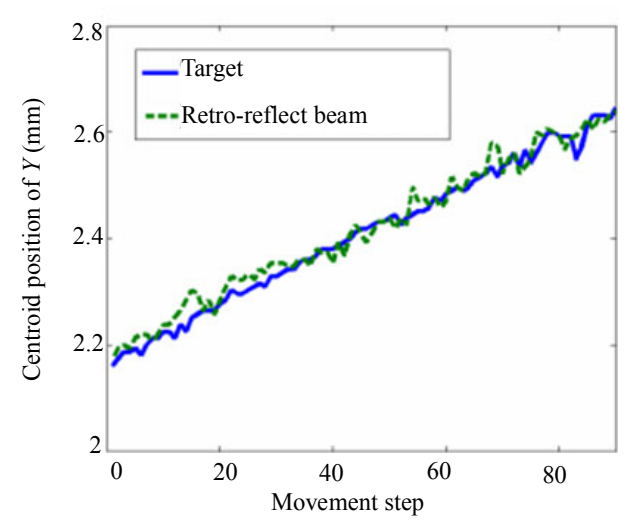

(b)

Fig. 7 Results of target moving along horizontal: (a) relationship between movement step and centroid position of $Y$ and (b) relationship between movement step and centroid position of $X$.

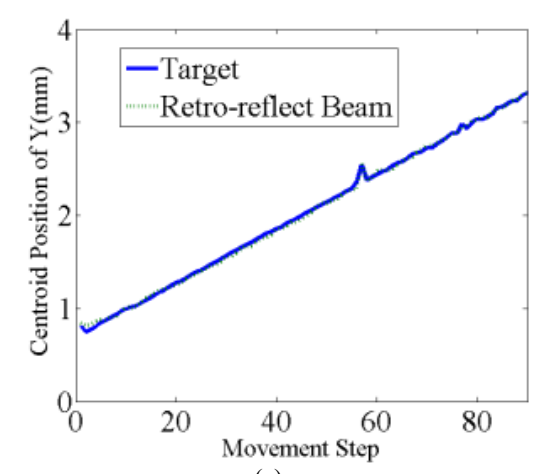

(a)

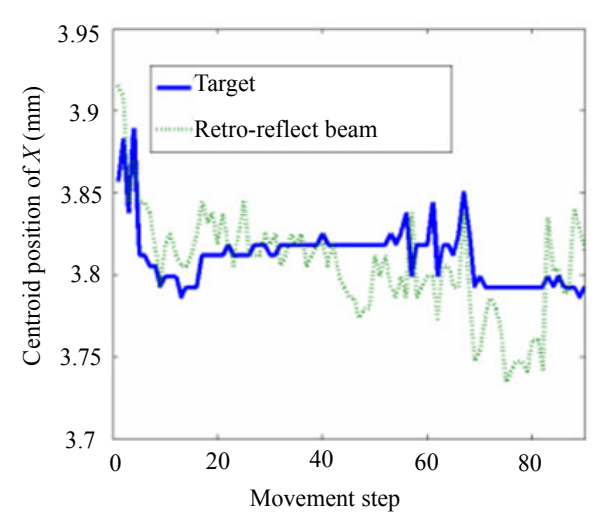

(b)

Fig. 8 Results of target moving along vertical: (a) relationship between movement step and centroid position of $Y$ and (b) relationship between movement step and centroid position of $X$.

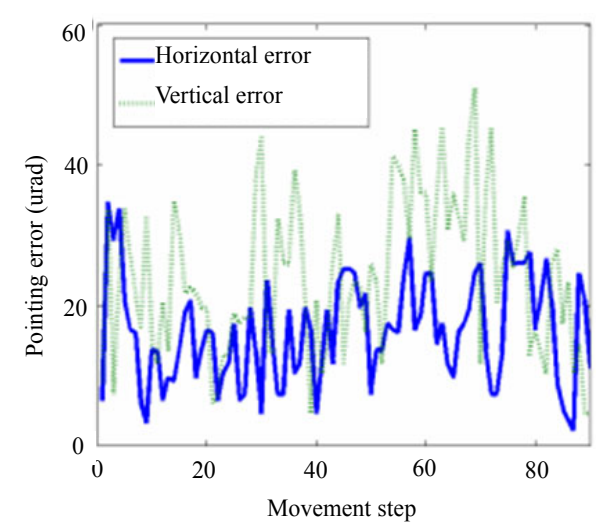

Fig. 9 Results of total pointing error.

Considering the composition of total pointing error, the beam steering method will cause pointing error, which is shown in Fig. 3, and it is mainly caused by the method itself and LC-OPA devices. On the other hand, errors coming from outer of the system will also contribute to the total pointing error. Factors, such as shape of the target, non-uniformity 
of the target surface reflectivity, spot size of the beam, background noise, and phase aberration, will cause the error of target location. So the total pointing error is shown in Fig. 9. After further calculation, an average pointing error of the LC-OPA aiming and pointing system is $19 \mu \mathrm{rad}$, and the max pointing error is $56 \mu \mathrm{rad}$.

In conclusion, a non-mechanical target aiming and pointing system with a single LC-OPA is demonstrated, and the LC-OPA based system has combined with an improved 2D beam steering method, and the feasibility of this method has been proved both in theory and experiment. A retro-reflect target is used to evaluate the aiming and pointing system. The system has a maximum FOV of $\pm 22 \mathrm{mrad}$ on the theory, and the average pointing error of the system is $19 \mu \mathrm{rad}$. The maximum pointing error is $56 \mu \mathrm{rad}$. Detailed error analysis of this system and tracking performance would be performed in the future work.

Open Access This article is distributed under the terms of the Creative Commons Attribution 4.0 International License (http://creativecommons.org/licese/by/4.0/), which permits unrestricted use, distribution, and reproduction in any medium, provided you give appropriate credit to the original author(s) and the source, provide a link to the Creative Commons license, and indicate if changes were made.

\section{References}

[1] C. R. Cooke, "Automatic laser tracking and ranging system," Applied Optics, 1972, 11(2): 277-284.

[2] P. M. Livingston, J. L. Jacoby, and W. S. Tierney, "Laser beam active tracking for specular objects to fractions of Lambda/D," Applied Optics, 1985, 24(13): 1919-1925.

[3] F. E. Hoge, "Integrated laser/radar satellite ranging and tracking system," Applied Optics, 1974, 13(10): 2352-2358.

[4] J. F. Riker, "Active tracking lasers for precision target stabilization,” Proc. SPIE, 2003, 5087: 1-12.

[5] J. E. Curtis, B. A. Koss, and D. G. Grier, "Dynamic holographic optical tweezers," Optics Communications, 2002, 207(1-6): 169-175.

[6] P. F. Mcmanamon, P. J. Bos, M. J. Escuti, J. Heikenfeld, S. Serati, H. Xie, et al., "A review of phased array steering for narrow-band electrooptical systems," Proceeding of the IEEE, 2009, 97(6): 1078-1096.

[7] E. Ha, L. Allard, L. Sjo, D. Engstro, S. Ha, Q. Wang, et al., "Retrocommunication utilizing electroabsorption modulators and nonmechanical beam steering," Optical Engineering, 2005, 44(4): 45001-1-45001-8.

[8] E. Haellstig, J. Stigwall, M. Lindgren, and L. Sjoqvist, "Laser beam steering and tracking using a liquid crystal spatial light modulator," Proc. SPIE, 2003, 5087: 13-23.

[9] D. Engström, J. Bengtsson, E. Eriksson, and M. Goksör, "Improved beam steering accuracy of a single beam with a 1D phase-only spatial light modulator," Optics Express, 2008, 16(22): 18275-18287.

[10] A. Linnenberger, S. Serati, and J. Stockley, "Advances in optical phased array technology," Proc. SPIE, 2006, 6304: $1-9$.

[11] L. Kong, Y. Zhu, Y. Song, and J. Yang, "Beam steering approach for high-precision spatial light modulators," Chinese Optics Letters, 2010, 8(11): 1085-1089. 